\title{
Evaluation of the Range Accuracy and the Radiometric Calibration of Multiple Terrestrial Laser Scanning Instruments for Data Interoperability
}

\author{
Kim Calders, Mathias I. Disney, John Armston, Andrew Burt, Benjamin Brede, \\ Niall Origo, Jasmine Muir, and Joanne Nightingale
}

\begin{abstract}
Terrestrial laser scanning (TLS) data provide 3-D measurements of vegetation structure and have the potential to support the calibration and validation of satellite and airborne sensors. The increasing range of different commercial and scientific TLS instruments holds challenges for data and instrument interoperability. Using data from various TLS sources will be critical to upscale study areas or compare data. In this paper, we provide a general framework to compare the interoperability of TLS instruments. We compare three TLS instruments that are the same make and model, the RIEGL VZ-400. We compare the range accuracy and evaluate the manufacturer's radiometric calibration for the uncalibrated return intensities. Our results show that the range accuracy between instruments is comparable and within the manufacturer's specifications. This means that the spatial XYZ data of different instruments can be combined into a single data set. Our findings demonstrate that radiometric calibration is instrument specific and needs to be carried out
\end{abstract}

Manuscript received April 22, 2016; revised October 17, 2016; accepted December 30, 2016. Date of publication February 17, 2017; date of current version March 17, 2017. This work was supported in part by the Metrology for Earth Observation and Climate Project (MetEOC-2) within the European Metrology Research Programme (EMRP) funded by the EMRP participating countries within EURAMET and the European Union under Grant ENV55, in part by the U.K. NERC National Centre for Earth Observation through funds for the purchase of the UCL RIEGL VZ-400 instrument and the Spectralon panels, and in part by TERN Auscover for funding the shipping of the panels to Australia. The work of B. Brede was supported by ESA-ESRIN through the IDEAS + project.

K. Calders is with the Earth Observation, Climate and Optical Group, National Physical Laboratory, Teddington, TW11 OLW, UK and the department of Geography, University College London, London WC1E 6BT, UK (contact: kim.calders@npl.co.uk; kim.calders@ucl.ac.uk)

M. Disney is with the Department of Geography, University College London, London WC1E 6BT, U.K., and also with the NERC National Centre for Earth Observation, U.K.

J. Armston was with the Queensland Department of Science, Information Technology and Innovation, Joint Remote Sensing Research Program, University of Queensland, Brisbane, QLD 4072, Australia. He is now with the Department of Geographical Sciences, University of Maryland, College Park, MD 20742 USA.

A. Burt is with the Department of Geography, University College London, London WC1E 6BT, U.K.

B. Brede is with the Laboratory of Geo-Information Science and Remote Sensing, Wageningen University, 6700 AA Wageningen, The Netherlands.

N. Origo and J. Nightingale are with the Earth Observation, Climate and Optical Group, National Physical Laboratory, Teddington TW11 OLW, U.K.

J. Muir was with the Queensland Department of Science, Information Technology and Innovation, Brisbane, QLD 4072, Australia. She is now with the Precision Agriculture Research Group, University of New England, Armidale, NSW 2351, Australia, and also with the Joint Remote Sensing Research Program, University of Queensland, Brisbane, QLD 4072, Australia.

Color versions of one or more of the figures in this paper are available online at http://ieeexplore.ieee.org.

Digital Object Identifier 10.1109/TGRS.2017.2652721 for each instrument individually before including reflectance information in TLS analysis. We show that the residuals between the calibrated reflectance panels and the apparent reflectance measured by the instrument are greatest for highest reflectance panels (residuals ranging from 0.058 to 0.312 ).

Index Terms-Data interoperability, radiometric calibration, RIEGL VZ-400, terrestrial light detection and ranging (LiDAR).

\section{INTRODUCTION}

$\mathbf{F}$ ORESTS account for approximately $31 \%$ of the land surface on the earth, covering a total area of approximately 4 billion hectares [1]. These forest ecosystems play vital productive, recreational, climatological, and ecological roles. Precise and accurate data about forest structure and function are needed to keep these roles balanced [2]. Decisions made by policy makers or natural resource managers require input data that are not linked with the spatial scales covered by conventional forest inventory methods. Remote sensing, in particular from satellite and airborne sensors, can obtain data over large or inaccessible forested areas. Such data are seen as one of the key ways to provide quantitative information on forests, particularly in developing countries [3]. A critical issue in using remote sensing data of any sort is interoperability, i.e., being able to combine observations from multiple sensors in a consistent way. This requires accurate characterization of the instrument's radiometric and geometric performance, ideally via cross calibration.

Light detection and ranging (LiDAR) is an active remote sensing method to assess forest structure directly by transmitting laser energy and analyzing the reflected energy as a function of time. Airborne LiDAR has been used in forest inventories since the 1980s [4], [5]. Terrestrial LiDAR has been used for finer scale forest measurements since the late 1990s, but uptake for operational forest monitoring has been slower [6]. Terrestrial LiDAR, also called terrestrial laser scanning (TLS), is a ground-based method that can measure the 3-D distribution of plant constituents to centimeter or even millimeter precision and accuracy at plot-level scales. There are two general modeling approaches for extracting forest attributes from TLS data: gap probability-based methods and geometric modeling methods [6]. Several studies in the last decade have demonstrated the use of TLS as a rapid and robust measurement tool for forest monitoring [7]-[13]. 
TABLE I

OVERVIEW OF THE RIEGL VZ-400 LASER SCANNERS USED IN THE EXPERIMENTS (RECORDED BEFORE RADIOMETRIC CALIBRATION)

\begin{tabular}{l|r|r|r}
\hline Owner & Year manufactured & Time power on & Time laser on \\
\hline $\mathrm{RSC}^{1}$ & 2012 & $2816 \mathrm{hrs}$ & $176 \mathrm{hrs}$ \\
$\mathrm{UCL}^{2}$ & 2014 & $179 \mathrm{hrs}$ & $33 \mathrm{hrs}$ \\
WUR $^{3}$ & 2010 & $1564 \mathrm{hrs}$ & $186 \mathrm{hrs}$ \\
\hline
\end{tabular}

${ }^{1}$ Queensland Government \& University of Queensland, Australia

2 University College London, United Kingdom

3 Wageningen University, The Netherlands

The availability of TLS instruments is expected to increase over the next years, offering new opportunities such as revisiting plots more frequently, acquiring more plots for the same investment, scanning larger areas [14], or providing more accurate comparisons of similar forest types in different biomes. However, the already increasing variety of instruments with different designs and capabilities means that data and instrument interoperability is a challenge that will need to be overcome to make the best use of TLS data from various sources.

In this paper, we intercompare the radiometric calibration of three TLS instruments. The instruments we compare here are the same make and model, the RIEGL VZ-400; however, the intercomparison approach we present is more generally applicable. The previous study [15] comparing the performance of different commercial instruments for forest monitoring suggested that at the time of writing, the RIEGL VZ-series offers the highest quality data for monitoring vegetation structure due to its multiple return output. We compare the range accuracy of the individual scanners and evaluate the manufacturer's radiometric calibration of the uncalibrated return intensities [16]. This work gives some insight into the potential for large-scale TLS mapping ( $>5-10 \mathrm{ha}$ ) using multiple instruments and their data interoperability. The calibrated intensity values allow us to relate these uncalibrated intensities to quantitative properties, namely, the reflectance of the target materials.

\section{MAterial And Methods}

\section{A. TLS Instruments}

In this paper, we compare three RIEGL VZ-400 terrestrial laser scanners (RIEGL Laser Measurement Systems GmbH, Horn, Austria). The individual scanner information prior to the comparison experiment is summarized in Table I, illustrating their different manufacturing dates and time in use.

The VZ-400 is a time-of-flight waveform recording scanner and has a range $(R)$ up to $350 \mathrm{~m}$. The arrival time of the return echo $\left(t_{i}\right)$ is used to calculate the range of individual targets [17]

$$
R_{i}=\frac{v_{g}}{2} \times\left(t_{i}-t_{\text {ref }}\right)
$$

where $v_{g}$ is the group velocity speed of light (about $3 \times 10^{8} \mathrm{~m} / \mathrm{s}$ ) and $t_{\text {ref }}$ is the timestamp of laser pulse emission. $v_{g}$ is defined as $c_{0} / n_{g}$, where $c_{0}$ is the speed of light in vacuum and the factor $n_{g}$ addresses the group velocity index of refraction, which is influenced by air temperature, air pressure, and humidity [18]. All RIEGL instruments are calibrated at well-defined atmospheric conditions at the manufacturer's facilities, and a detailed description of atmospheric parameter effects on $v_{g}$ is given in [18]. The evaluation of atmospheric effects on $v_{g}$ is outside the scope of this paper, but the influence is expected to be minimal at the relatively short range values recorded in forests [19].

The line scanning mechanism uses a rotating multifacet polygonal mirror, which leads to fully linear, unidirectional, and parallel scan lines. A collimated beam is used [20] and the beam divergence is nominally $0.35 \mathrm{mrad}$. The scanner operates at a wavelength of $1550 \mathrm{~nm}$ (near infrared) [21]. The RIEGL VZ-400 scanner measures up to four returns per emitted pulse, with returns being derived from onboard waveform processing. The advantage of having multiple returns in vegetation studies has been discussed in [11] and [22]. These studies revealed a weaker response at greater canopy height from single return instruments, concluding that multiple returns will lead to an improved vertical sampling of vegetation. The onboard waveform processing of the RIEGL VZ-400 instrument allows for echo digitization: the system samples signals every $2 \mathrm{~ns}$ and converts them into a digital representation before target detection. This is an advantage compared with analog discrete return systems, which have to accomplish target detection in real time using analog electronics. The latter can be problematic when return energy from different targets overlaps and ranging errors occur for second and higher order returns [20]. The RIEGL VZ-400 only records samples (i.e., sampling blocks) of the returned waveform and [20] refers to the VZ-400 data as "tightly coupled echo signal samples."

RIEGL VZ-400 instruments provide range, scan angles, calibrated amplitude, apparent reflectance, and pulse deviation for each triggered return. The calibrated amplitude is proportional to a fixed device-specific echo signal power level (e.g., the detection limit of the device). The calibrated amplitude for each measurement is $A_{\mathrm{dB}}$

$$
A_{\mathrm{dB}}=10 \times \log \left(\frac{P_{\mathrm{echo}}}{P_{\mathrm{DL}}}\right) .
$$

Here, $P_{\text {echo }}$ is the optical input power for a specific measurement and $P_{\mathrm{DL}}$ is the minimum detectable input power [16]. RIEGL performs amplitude calibration by varying the $P_{\text {echo }}$ over its dynamic range using different calibration targets and storing the device's internal uncalibrated amplitude. $A_{\mathrm{dB}}$ is range dependent, and therefore, the apparent reflectance $\rho_{\text {app }}$ is a more useful measure when combining scan data from different instruments or scan locations. $\rho_{\text {app }}$ is defined as the reflectance of a diffuse Lambertian target filling the laser beam that would return the same amount of intensity as the actual target at the same distance [8]. RIEGL derives $\rho_{\text {app, dB }}$ values from subtracting the reference-range-dependent $A_{\mathrm{dB}, \text { ref }}(R)$ from the target's actual $A_{\mathrm{dB}}$. The reference is the received reflectance from a nearly $100 \%$ reflective extended Lambertian target orthogonal to the direction of the laser beam. The peak $A_{\mathrm{dB}}$ of the VZ-400 is observed around $7 \mathrm{~m}$, and the laser range $1 / R^{2}$ law is applicable to $A_{\mathrm{dB}}$ from distances further than $20 \mathrm{~m} \mathrm{[16].}$ 

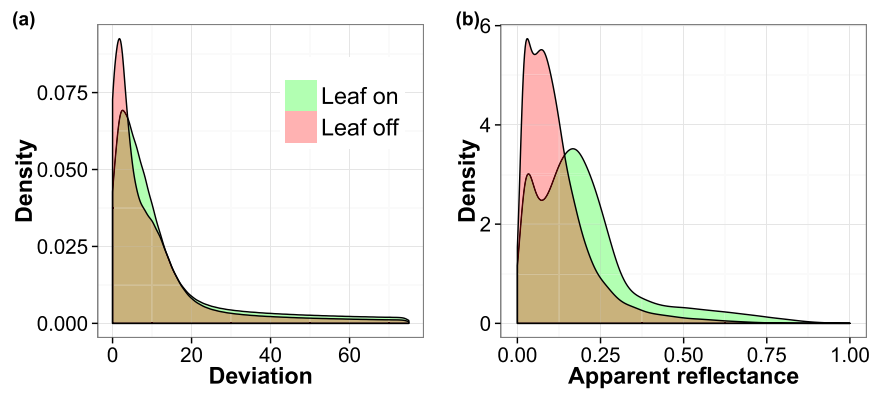

Fig. 1. Density plot illustrating a typical dynamic range of deviation and $\rho_{\text {app }}$ values recorded in a deciduous woodland, Wytham Woods, U.K. [14]. Data were captured with the UCL RIEGL VZ-400 in 2015 from the same scan location in (a) summer (leaf-on) and (b) winter (leaf-off).

Gaussian decomposition, the reconstruction of a waveform pulse using one or more Gaussian pulses is common practice in LiDAR analysis [23]. The problem with this method is that the Gaussian model generally does not fit the entire dynamic range of the system equally well and this can lead to a ranging error of several millimetres, especially for highamplitude signals. Therefore, the RIEGL VZ-400 onboard waveform processing uses stored device-specific pulse shapes that cover the entire dynamic behavior of the system and a 2-D optimization algorithm [16]. The comparison of the recorded waveform with the stored reference shapes is quantified by the pulse deviation $(\delta)$ and this value increases for overlapping pulses or slanted targets

$$
\delta=\sum_{i=1}^{N}\left|s_{i}-p_{i}\right|
$$

where $N$ is the total number of digitized samples $s_{i}$ that are matched to the corresponding reference pulse, which has interpolated $p_{i}$ values for the phase and amplitude given by the optimization algorithm. Fig. 1 gives an example of a typical deviation and $\rho_{\text {app }}$ dynamic range of a deciduous woodland in the leaf-on (summer) and leaf-off (winter) conditions.

\section{B. Range Accuracy}

RIEGL defines accuracy as the degree of conformity of a measured quantity to its actual (true) value [21]. Range accuracy is derived from the calibration certificates provided by RIEGL for a number of different ranges and targets. These certificates show nominal and measured range values to fixed targets. The nominal values are measured at certain time intervals using a total station. The nominal values on the certificates are slightly different between different instruments. This is because the calibration measurements are done in a fixed setup that allows multiple scanners to be mounted at once. Therefore, depending on which position the scanner was mounted, the nominal values on the calibration certificate can differ slightly. The nominal values of the targets in the fixed setup are measured at certain time intervals with a total station (RIEGL, personal communication).

\section{Experimental Setup for Evaluating the Radiometric Calibration}

Radiometric calibration is described as the process of deriving physically well-defined radiometric quantities from an instrument's raw measurements [24]. A radiometric calibration for terrestrial laser scanners is typically based on a set of measurements using multiple calibrated reflectance panels at different distances [25], [26], and Hartzell et al. [27] used this approach to generate an empirical radiometric VZ-400 calibration model.

We used five different SphereOptics (SphereOptics $\mathrm{GmbH}$, Germany) reflectance panels of dimensions $20 \mathrm{~cm} \times 20 \mathrm{~cm}$ with the nominal reflectance values $0.05,0.2$, $0.5,0.9$, and 0.95 . The calibration report of each panel (dated March 10, 2015) shows that the total diffuse hemispherical reflectances of these panels are $0.047,0.242$, $0.418,0.902$, and 0.947 at wavelength $1550 \mathrm{~nm}$, calibrated traceable to a Physikalisch-Technische Bundesanstalt reference standard with a total calibration uncertainty of $\pm 0.35 \%$ (95\% confidence).

The same reflectance panels were scanned in May 2015 (UCL, WUR, London, U.K.) and August 2015 (RSC, Brisbane, Australia). All experiments were conducted indoors and all panels were scanned with a $0.04^{\circ}$ angular resolution. The scanners and panels were mounted on a tripod and leveled using a spirit level. Each panel was scanned from six nominal distances: $1 \mathrm{~m}, 2 \mathrm{~m}, 5 \mathrm{~m}, 10 \mathrm{~m}, 20 \mathrm{~m}$, and $30 \mathrm{~m}$ (RSC) or $37.5 \mathrm{~m}$ (UCL, WUR). The nominal distances were measured using a measurement tape, but the VZ-400 range recordings are used in the further analysis. The RIEGL VZ-400 supported minimum range is $1.5 \mathrm{~m}$ [21], but it is possible to reduce this to $0.5 \mathrm{~m}$. The maximum range of this experiment is sufficient for application in a range of forest types as the bulk of the LiDAR returns in these ecosystems are generally close to the scanner. The previous work [11] demonstrated that for five different forest types in Australia (ranging from woodland to rainforest), the 90th percentile of the return frequency distribution ranges between 9 and $46 \mathrm{~m}$.

To compare the instrument's apparent reflectance measurements with the calibrated panel reflectance values, we converted the $\rho_{\mathrm{app}, \mathrm{dB}}$ readings from the scanner into a bidirectional reflectance factor $\rho_{\text {app }}$ using

$$
\rho_{\text {app }}=10^{\rho_{\text {app }, \mathrm{dB}} / 10} \text {. }
$$

\section{RESUlTS AND DiscUSSION}

The number of returns per panel varied from more than 69000 at a $1-\mathrm{m}$ distance (Fig. 2) to only 21 to 33 returns for the panels at $37.5 \mathrm{~m}$. The angular range was largest for the panel at $1 \mathrm{~m}$ (approximately $10^{\circ}-11^{\circ}$, which equates to a maximum angle of incidence of approximately $5^{\circ}-5.5^{\circ}$ ) and reduced with increasing panel-instrument distance (e.g., less than $0.5^{\circ}$ at $20 \mathrm{~m}$ ). The attributes of returned laser pulses are determined by the distance, beam divergence, and angle of incidence. A nonperpendicular angle of incidence leads to the broadening of the return pulse. Pfennigbauer et al. [28] demonstrated the weak effect of pulse broadening for the RIEGL VZ-instrument, mainly due to the small beam divergence of the instrument (nominally $0.35 \mathrm{mrad}$ ). They stated that it was questionable whether an increase in pulse width was measurable for incidence angles below $5^{\circ}$. These findings were confirmed in [29], which reported no real differences for incidence angles smaller than $20^{\circ}$ at distances smaller 
(a)

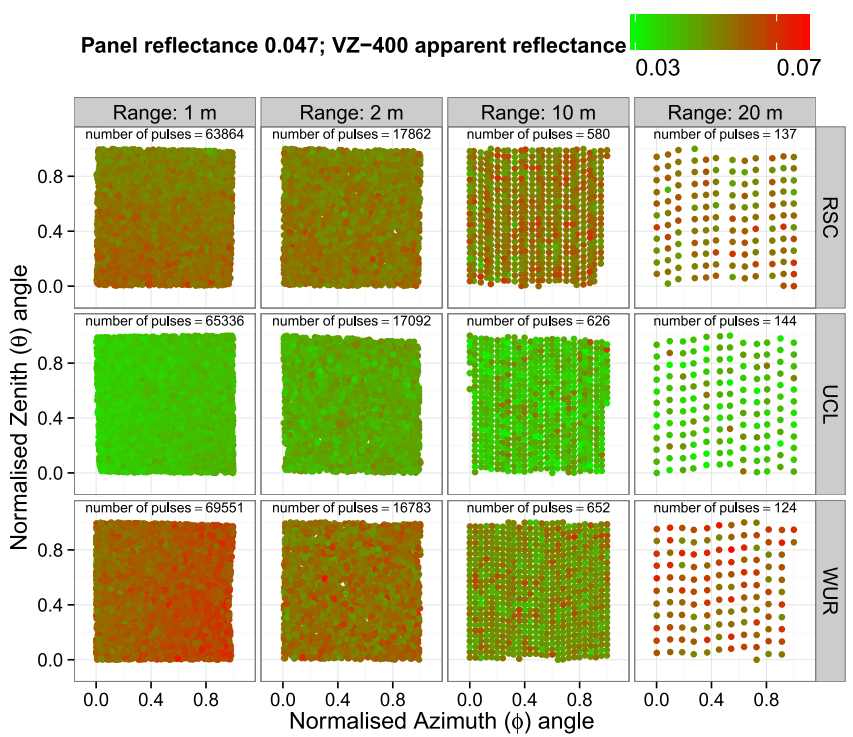

(c)

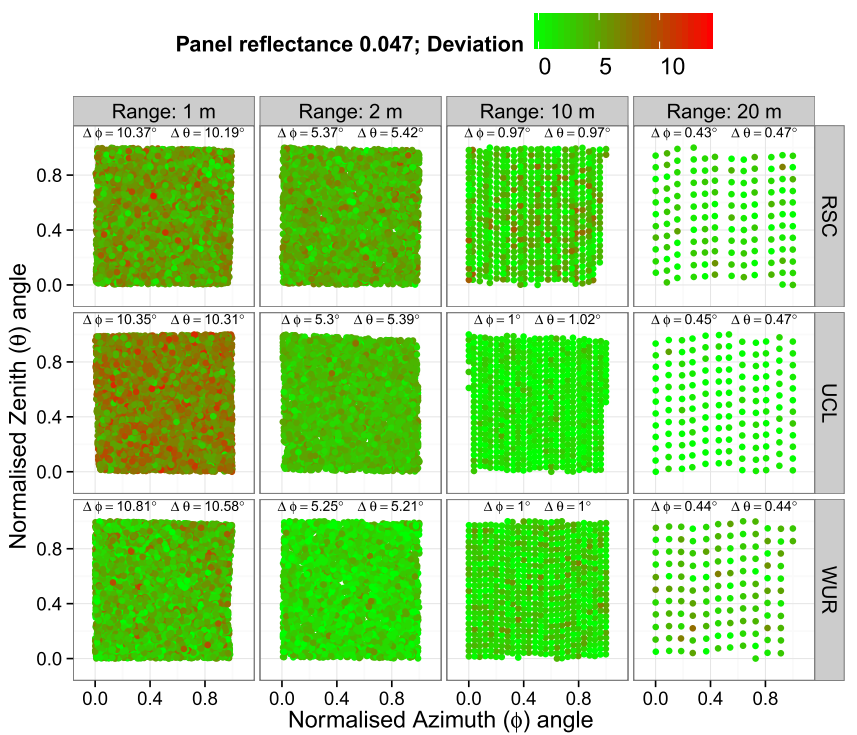

(b)

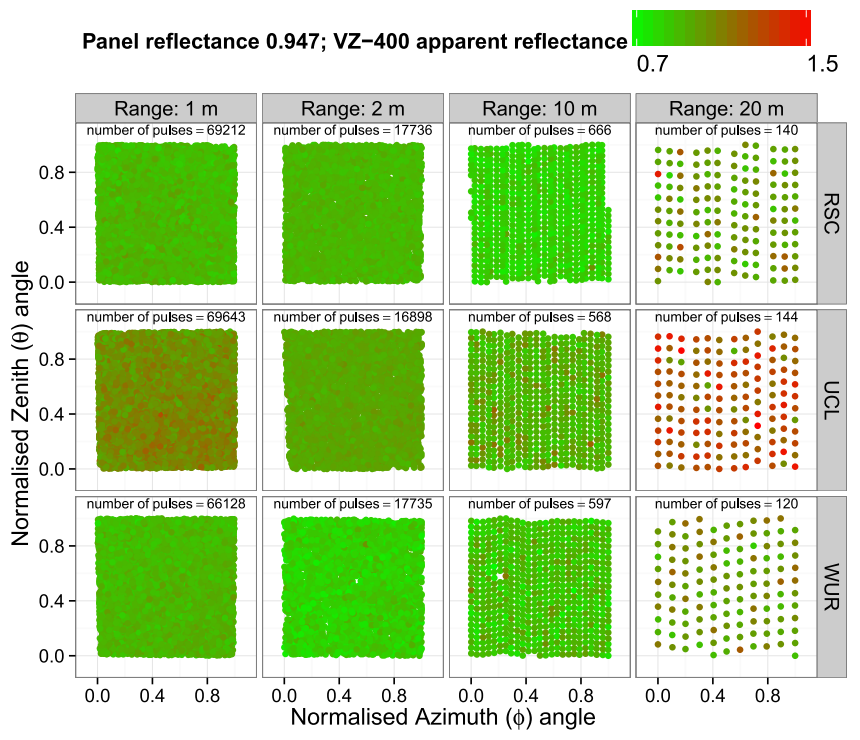

(d)

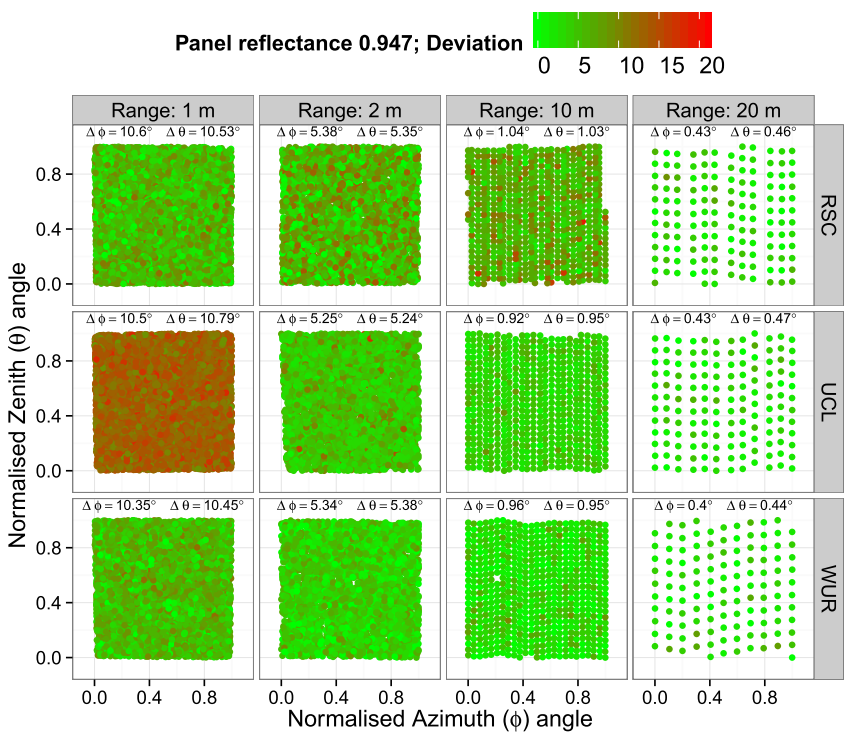

Fig. 2. Examples of the distribution of VZ-400 $\rho_{\text {app }}$ and deviation values on the 0.047 and 0.947 reflectance panels for the distances of $1,2,10$, and $20 \mathrm{~m}$. $\Delta \phi$ and $\Delta \Theta$ give the true angular azimuth and zenith angle intervals, respectively, that cover the panel.

than $32 \mathrm{~m}$. Therefore, we average point attributes per panel similar to [25] and [26]. Fig. 2 shows the distribution of the returns on the 0.047 and 0.947 reflectance panels for the distances of $1,2,10$, and $20 \mathrm{~m}$ and their corresponding $\rho_{\text {app }}$ and deviation values. The residuals around the mean value are shown in Fig. 3. All panels show a homogeneous distribution of pulse attributes across the panel. Fig. 3(a) suggests that the lowest reflectance panel (0.047) at close range has a weak spatial relation of the $\rho_{\text {app }}$ residuals. However, the panel at $2 \mathrm{~m}$ and all panels at further distances do not show similar patterns. We suggest that this is due to the low signal-to-noise at short distances and this might be part of the reason why the RIEGL VZ-400 supported minimum range is $1.5 \mathrm{~m}$ [21].

\section{A. Raw Waveform Analysis}

Fig. 4(a) shows typical raw waveforms recorded by the VZ-400. Similar to [27], an offset between the peak in waveform intensity and the registered range is observed. This range offset has previously been observed in the full waveform airborne RIEGL LMS-Q680I sensor and was attributed to the transimpedance amplifier that is used in the receiver electronics to exhibit sufficient nonlinearity to introduce a significant delay that depends on the recorded intensity samples [30]. RIEGL [17] confirms that this is also the case for the VZ-400 sensor and a calibration table (proprietary information) is used to compensate for the range deviation.

Stronger returns, i.e., higher panel reflectance, cause the typical VZ-400 long trailing edge to be more prominent than when the return energy is lower [Fig. 4(a)] due to higher energy returns causing nonlinearity in the detector electronics. This is observed for all three instruments and consistent with [27] and [23].

The waveforms clearly show the different dynamic ranges of the digitizer intensity values of the three scanners. For similar 
(a)

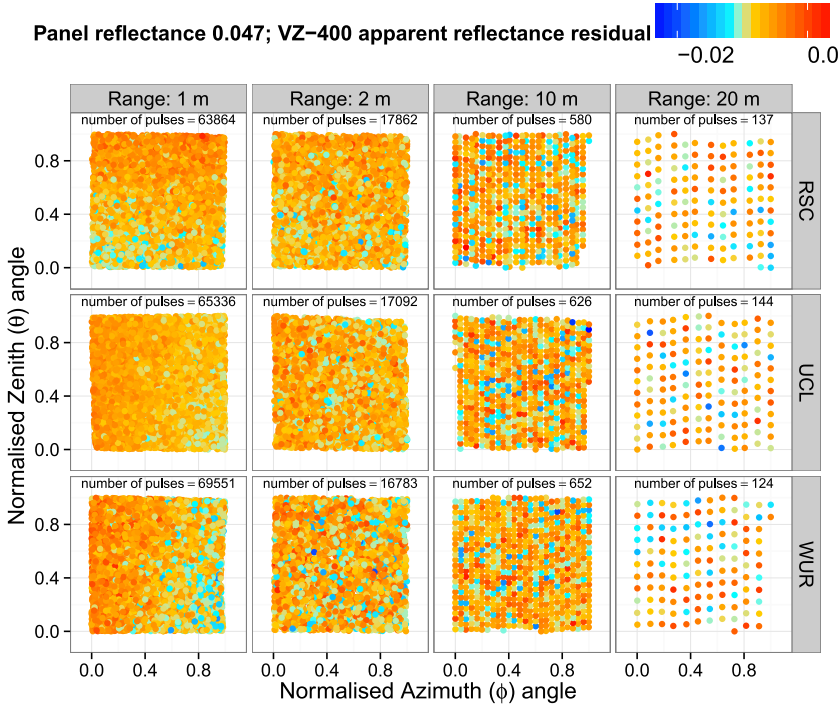

(c)

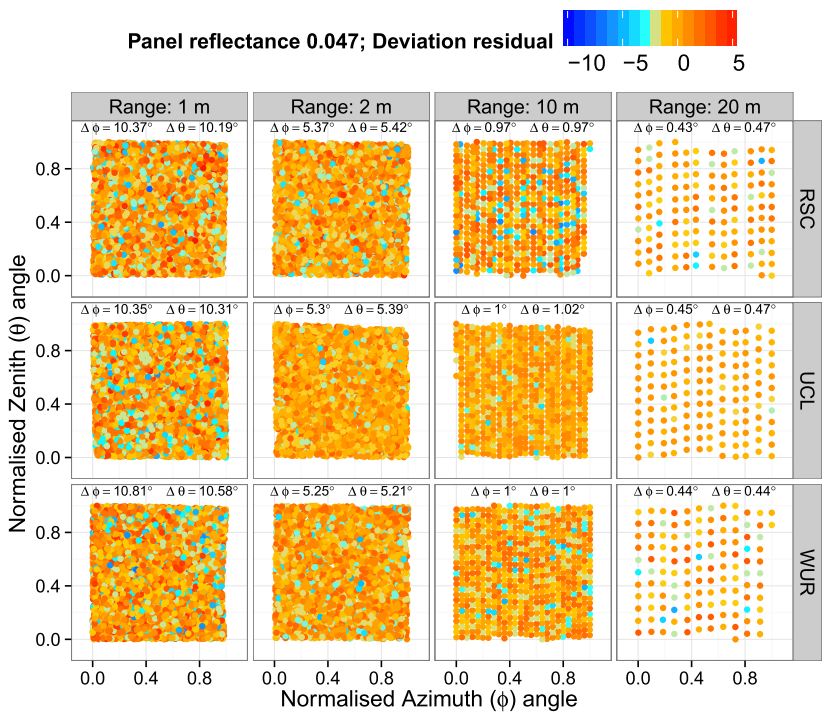

(b)
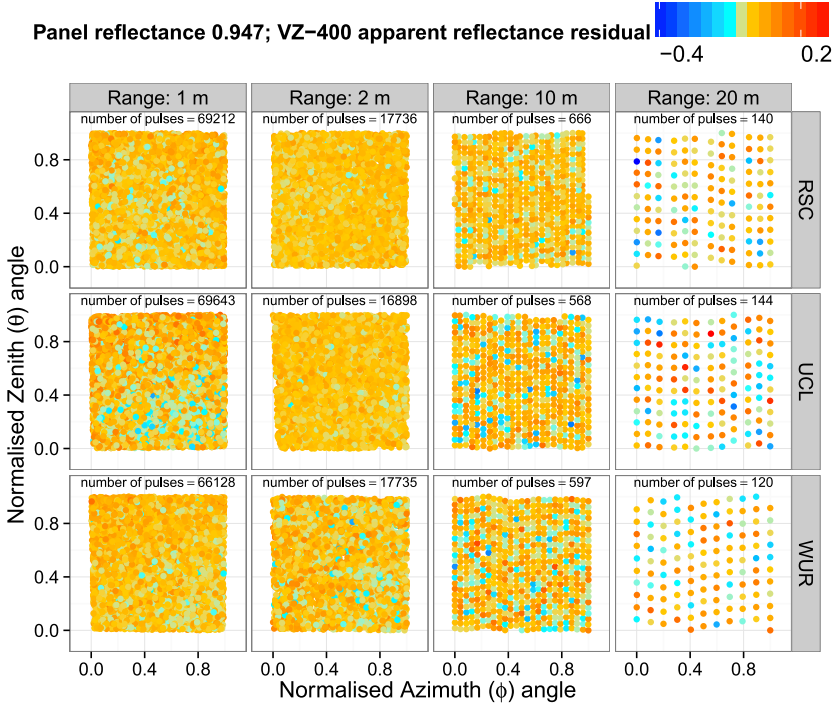

(d)

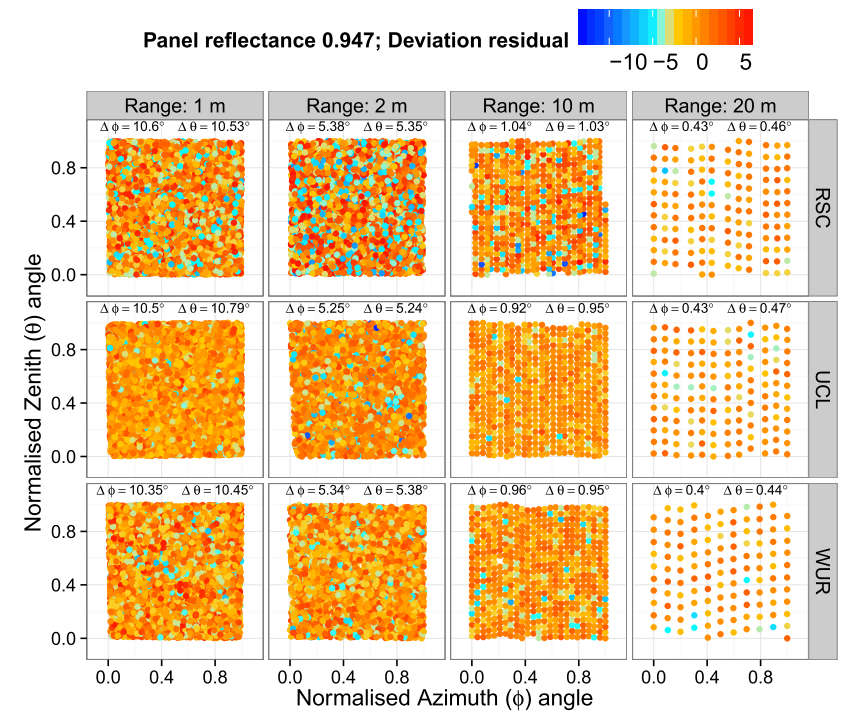

Fig. 3. Examples of the distribution of VZ-400 $\rho_{\mathrm{app}}$ and deviation residuals (around the mean value) on the 0.047 and 0.947 reflectance panels for the distances of $1,2,10$, and $20 \mathrm{~m} . \Delta \phi$ and $\Delta \Theta$ give the true angular azimuth and zenith angle intervals, respectively, that cover the panel.

measurements (i.e., the same reflectance panel and distance), the RSC instrument records the highest absolute intensity samples. The differences in waveform length [Fig. 4(a)] are due to the constant development of the production procedure and the manufacturer has reduced the storage time of the waveform in newer (see Table I) instruments (RIEGL, personal communication). The relationship between maximum intensity sample (i.e., the waveform peak) and the panel reflectance is nonlinear. Fig. 4(b) shows that all instruments have the highest maximum intensity sample at $10 \mathrm{~m}$ for all the spectral panels and the lowest maximum intensity sample for the furthest panel distance. These observations agree with [16] evaluating this instrument and the nonlinear characteristics for distances up to about $20 \mathrm{~m}$ are due to vignetting or central obscuration.

\section{B. Range Accuracy}

Table II summarizes 20 different range accuracy assessments ranging from approximately 11.5 to $138 \mathrm{~m}$ (eight for the RSC and WUR instrument and four for the UCL scanner; RIEGL provides new calibration certificates after every service). All the differences between the instrument and nominal ranges lie within the manufacturer's specifications, which are the key for data interoperability. Most of the current studies and applications in TLS that use calibrated commercial instruments use only the spatial component of the data [6]. For example, vertical plant profiles describe the plant area volume density as a function of canopy height through estimates of the vertically resolved gap probability [11], [12], [31]. Other applications try to explicitly model the 3-D structure of vegetation, using the point clouds as input [13], [32][34]. These findings essentially mean that the spatial point cloud data (i.e., the XYZ data) of multiple VZ-400 instruments can be combined and used in one single data set. Using multiple instruments would significantly reduce the time spent in the field if using the same fieldwork protocol, allow larger areas to be scanned within the 
(a)

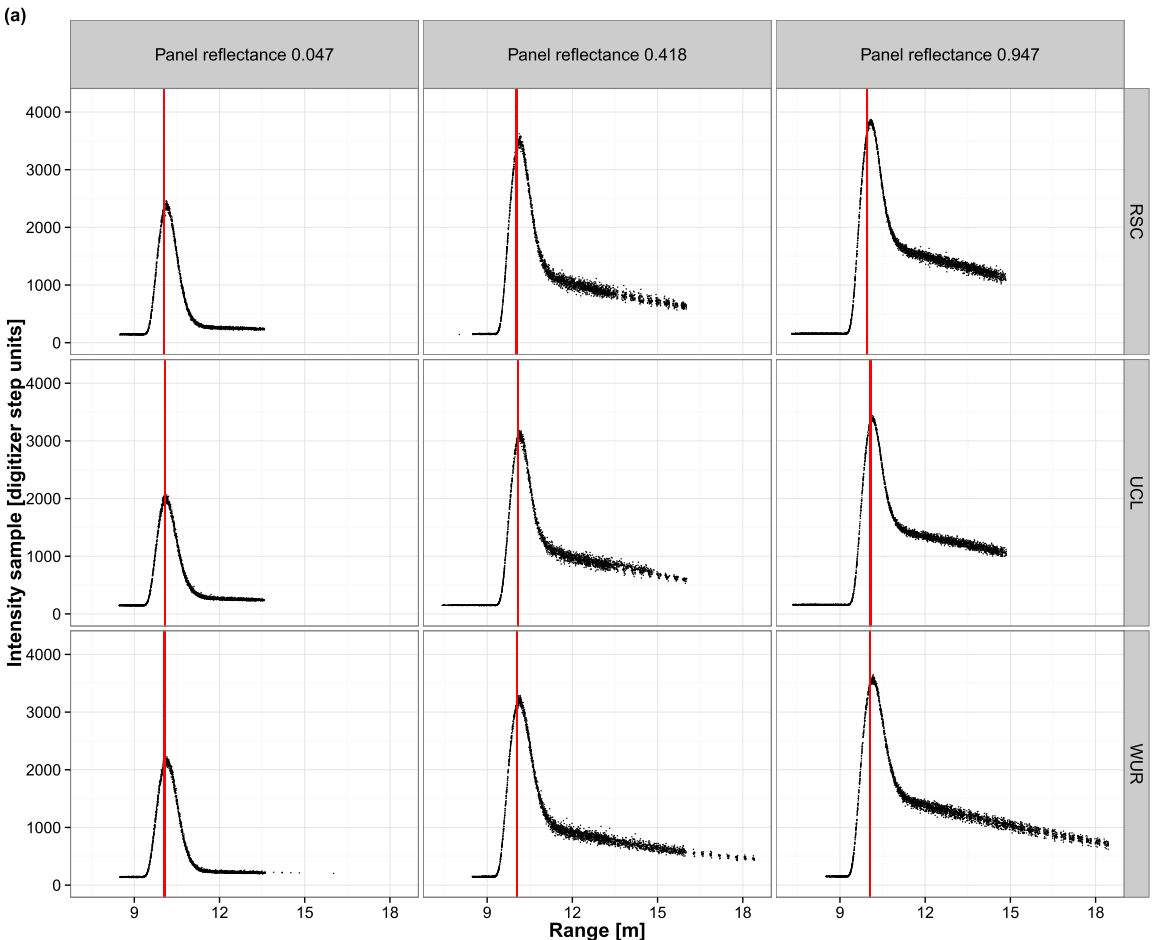

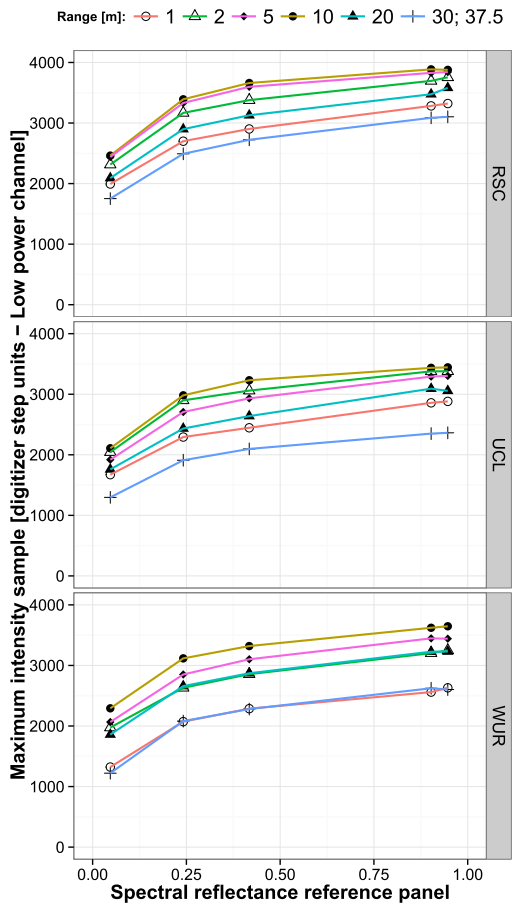

Fig. 4. RIEGL VZ-400 waveforms. (a) Examples of all raw waveforms for three different reflectance panels at a 10-m range. The vertical red line indicates the range registered by the instrument. (b) Overview of the maximum intensity sample (i.e., peak waveform) for all panels at all distances.

TABLE II

RANGE ACCURACY AsSESSMENT For DiFFERENT TARgET TyPES: RANGE Difference Between the MEasured Range With the RIEGL VZ-400 AND THE NOMINAL REFERENCE VALUES

\begin{tabular}{l|l|r|r|r}
\hline Target & Scanner & Nominal range [m] & $\Delta$ range $^{1}[\mathbf{m}]$ & Specified accuracy $\left.\mathbf{~}^{2} \mathbf{m}\right]$ \\
\hline Reflector foil & WUR & 13.771 & 0.002 & \pm 0.005 \\
(diameter 50mm) & WUR & 11.562 & -0.002 & \pm 0.005 \\
& RSC & 11.760 & 0.000 & \pm 0.005 \\
& RSC & 11.563 & 0.001 & \pm 0.005 \\
& UCL & 11.812 & 0.002 & \pm 0.005 \\
\hline Reflector foil & WUR & 136.909 & 0.001 & \pm 0.007 \\
(diameter 150mm) & WUR & 137.276 & -0.004 & \pm 0.007 \\
& RSC & 138.055 & 0.000 & \pm 0.007 \\
& RSC & 137.274 & 0.000 & \pm 0.007 \\
& UCL & 136.905 & 0.003 & \pm 0.007 \\
\hline Target plate & WUR & 131.330 & 0.001 & \pm 0.006 \\
$(\rho \geq 10 \%)$ & WUR & 131.324 & -0.002 & \pm 0.006 \\
& RSC & 131.330 & -0.003 & \pm 0.006 \\
& RSC & 102.320 & -0.002 & \pm 0.006 \\
& UCL & 131.324 & -0.001 & \pm 0.006 \\
\hline Target plate & WUR & 129.260 & 0.001 & \pm 0.006 \\
$(\rho \geq 80 \%)$ & WUR & 129.257 & -0.001 & \pm 0.006 \\
& RSC & 129.260 & -0.002 & \pm 0.006 \\
& RSC & 102.270 & -0.002 & \pm 0.006 \\
& UCL & 129.257 & 0.001 & \pm 0.006 \\
\hline
\end{tabular}

$1 \Delta$ range $=$ VZ-400 instrument value - nominal value

2 Manufacturer's accuracy specifications

same time frame or increase the number of scan locations within a plot.

\section{Evaluation of the Radiometric Calibration}

Fig. 5 compares the VZ-400 mean $\rho_{\text {app }}$ estimates with the reflectance values from the reference panels. Fig. 5 shows that $\rho_{\text {app }}$ estimates for higher reflectance targets generally have greater absolute bias and uncertainty. This is most prominent for the UCL scanner for the 0.902 and 0.947 reflectance panels at $20 \mathrm{~m}$, with the average overestimations of 0.254 and 0.312 , respectively (compared with 0.093 and 0.069 for the WUR instrument and 0.074 and 0.058 for the RSC instrument). In this scenario, the $\rho_{\text {app }}$ values are larger than 1 , which generally indicates a target reflecting with a higher reflectivity than that of a Lambertian target [16]. The larger uncertainty in RIEGL $\rho_{\text {app }}$ for higher reflectance targets is possibly caused by the $\log$ scale $(\mathrm{dB})$ recording of intensity data and the user should be extra careful about high reflectance values when carrying out a radiometric calibration. However, the majority of $\rho_{\text {app }}$ values measured in the field (see Fig. 1) are generally below 0.4 with a peak around approximately $0.05-0.1$ in leaf-off conditions and a bimodal distribution in leaf-on conditions (peaks approximately around 0.05 and 0.2 ). The average absolute $\rho_{\text {app }}$ residual over the 30 different scenarios was 0.074 for the UCL instrument, 0.05 for the WUR instrument, and 0.049 for the RSC instrument. The maximum and minimum absolute $\rho_{\text {app }}$ residuals were 0.312 (0.947 reflectance panel at $20 \mathrm{~m})$ and 0.001 ( 0.047 reflectance panel at $2 \mathrm{~m}$ ) for the UCL scanner, 0.164 (0.947 reflectance panel at $5 \mathrm{~m})$ and $0.001(0.418$ reflectance panel at $5 \mathrm{~m})$ for the WUR scanner, and 0.132 ( 0.947 reflectance panel at $10 \mathrm{~m})$ and $0(0.947$ reflectance panel at $30 \mathrm{~m})$ for the RSC scanner.

Although many TLS applications currently use XYZ point cloud data only (see Section III-B), inclusion of reflectance information or other attributes derived from raw waveforms may improve existing methods. The vertical plant profiles generated in [11] and [12] use the multiple return VZ-400 data 


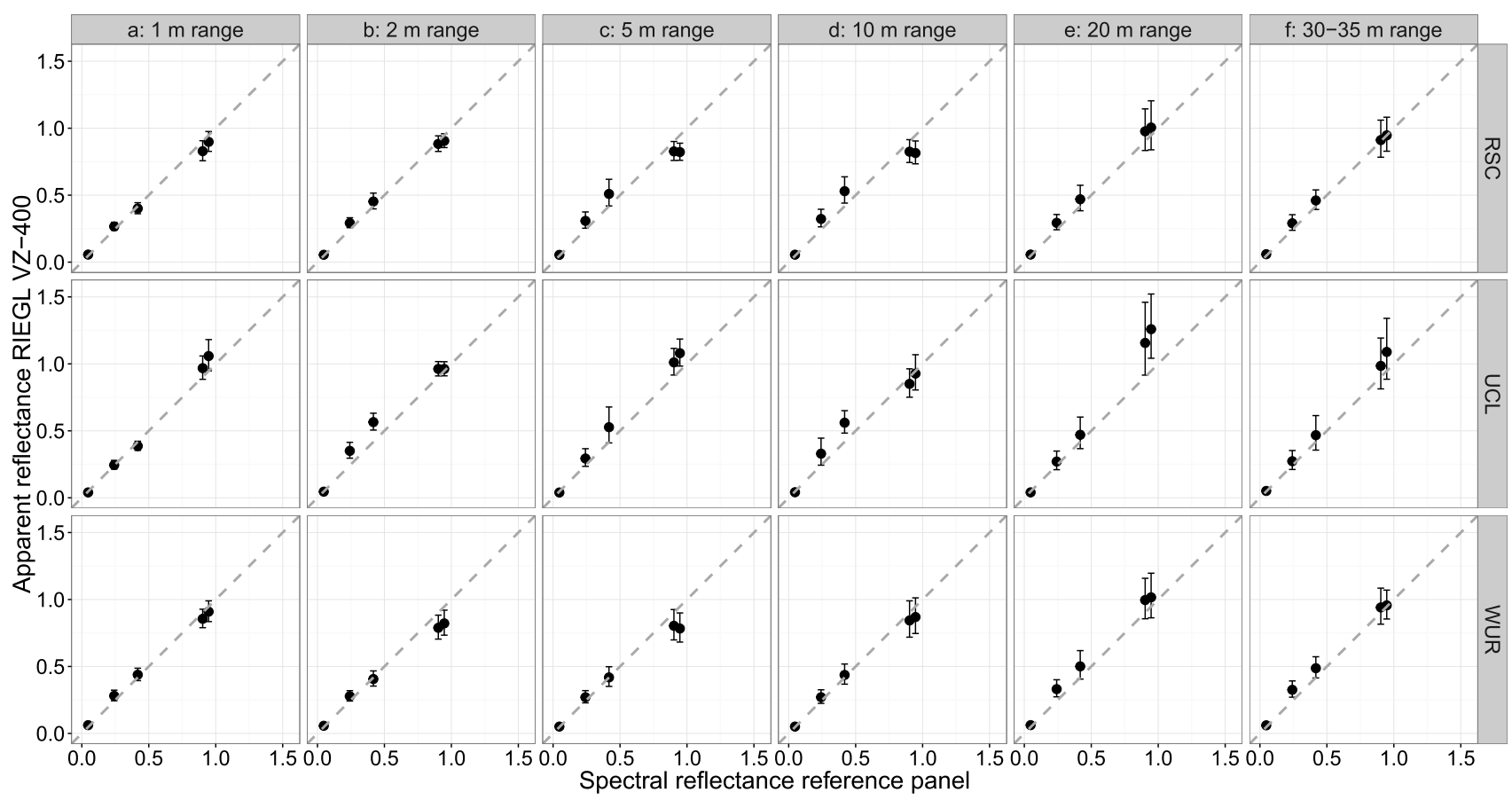

Fig. 5. Comparison of spectral reflectance from the reference panel with $\rho_{\text {app }}$ values measured by the VZ-400 for different ranges. The vertical error bars indicate the $95 \%$ confidence interval around the mean $\rho_{\text {app }}$ of the panel.

to estimate gap probability. Their method assumes that for a single outgoing pulse, each return equates to $1 / \mathrm{n}$ of the intercepted beam area, where $n$ is the total number of returns for that outgoing pulse. The use of additional $\rho_{\text {app }}$ information may further improve the estimation of intercepted beam area for each return [35]. Separating woody and nonwoody components in the point cloud is important to separate plant area profiles into leaf area profiles and wood area profiles. The distinct wood and leaf area profiles will provide more valuable information about ecosystem dynamics than the integrated plant area profiles. A separation of the returns is also important for geometric modeling, i.e., extracting the size and shape of tree components from point clouds. Aboveground biomass (AGB) is an important indicator for carbon storage and sequestration of forests and their productivity [36]. Nondestructive estimates of AGB using TLS data show significant differences from AGB estimates using traditional allometric equations [13]. The TLS method could be further improved if leafy and woody returns could be reliably separated, because leaves generally do not resemble the geometric structure of a cylinder. It is important to note that the RIEGL conversion of the calibrated amplitude $A_{\mathrm{dB}}$ (2) into $\rho_{\text {app }}$ applies only to targets that intercept the laser pulse completely (i.e., single returns) [16]. This complicates the use of intensity for multiple return LiDAR systems, because the intercepted laser energy from previous returns has to be accounted for.

The results in this paper indicate that the three VZ-400 sensors show different responses to the same reference panel at the same distance. This suggests that radiometric calibration models of one instrument cannot be used for another instrument. For example, Fig. 6 shows the spectral reflectance for bark and leaf in a deciduous woodland in the U.K. At $1550 \mathrm{~nm}$, the wavelengths of the RIEGL VZ-400 with

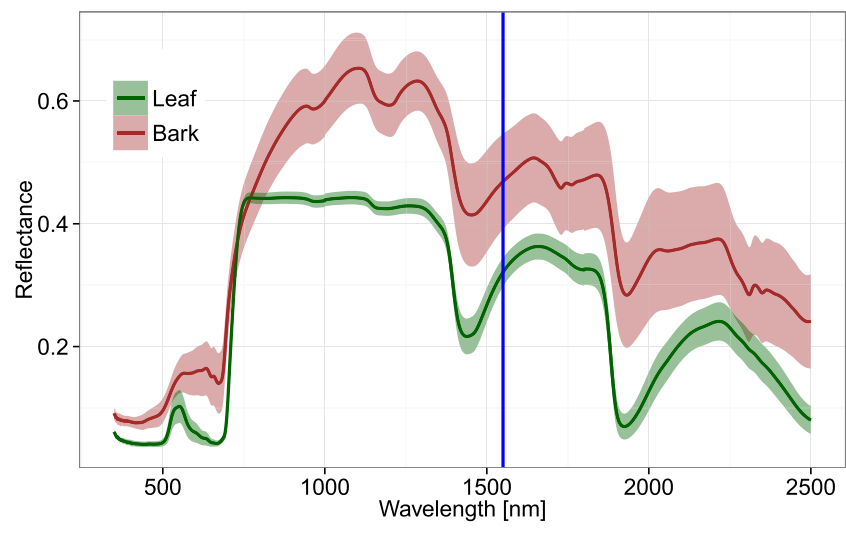

Fig. 6. Leaf and bark spectral reflectances of a deciduous woodland, Wytham Woods, U.K. The solid line indicates the weighted average spectral reflectance and the area around the mean represents the $95 \%$ coverage interval. The weights are allocated based on the number of trees per species, with Acer pseudoplatanus (55\%), Fraxinus excelsior (20.6\%), and Corylus avellana $(14.3 \%)$ being the dominating species. Data were captured with an ASD spectrometer in the summer of 2015. The vertical blue line represents the wavelength of the RIEGL VZ-400 $(1550 \mathrm{~nm})$.

the 95\% coverage interval of bark and leaf reflectance are $0.391-0.546$ and $0.298-0.344$, respectively. This suggests that for this particular forest, only a maximum calibration uncertainty of $4.7 \%$ absolute reflectance is acceptable in applications that benefit from the separation between woody and nonwoody components in the LiDAR data. However, using intensity information from a single wavelength scanner limits this separation because the received intensity is a function of the spectral reflectance properties of the canopy constituents, the area of the beam that is intercepted, and the local angle of incidence [37]. The dual-wavelength scanners DWEL and SALCA try to overcome the limitations of a single-wavelength 
scanner by taking the spectral ratio of the two laser wavelengths at approximately 1064 and $1550 \mathrm{~nm}$ [37], [38].

\section{CONCLUSION}

One of the challenges of interoperability is the commercial confidentiality of instrument internal performance properties, making interoperability difficult. This implies that it is generally up to the user to carry out calibration measurements. Our findings demonstrated that the XYZ data of VZ-400 sensors is intercomparable and we suggest that data from multiple instruments can be merged into a single data set. This enables us to use TLS in large-scale ( $>5-10 \mathrm{ha}$ ) mapping and merge data from different instruments together. An evaluation of the radiometric calibration of three same make and model sensors showed that radiometric calibrations and therefore $\rho_{\text {app }}$ are instrument specific and that absolute bias is greater for high reflectance returns. We therefore recommend a radiometric calibration of each instrument before including reflectance information in new or existing methods. To extend this paper to intercomparing instruments with different wavelengths and specifications, we would need to understand how the observed bias (and variance) might propagate through to derived quantities using a combination of measurement [39] and simulation [23].

3-D information plays an important role in the calibration and validation of many airborne and spaceborne sensors. This is particularly true of sensors that measure canopy structure near directly. A new large footprint spaceborne instrument, Global Ecosystem Dynamics Investigation (GEDI) LiDAR, is currently being developed and was scheduled for launch in 2018 [40]. The European Space Agency BIOMASS mission is expected to launch in 2020 and this spaceborne P-band RADAR system will acquire data at a spatial resolution of $200 \mathrm{~m}$ [41]. The combination of explicit 3-D forest stand reconstructions based on TLS data and radiative transfer models will provide a framework for testing the structural metrics derived from planned missions such as GEDI and BIOMASS.

\section{ACKNOWLEDGMENT}

The authors would like to thank the NERC Field Spectroscopy Facility for the help in advising on the provision and use of the Spectralon panels and P. Green and N. Fox for their helpful comments on this paper.

\section{REFERENCES}

[1] Global Forest Resources Assessment 2010, Main Report, FAO Forestry Paper 163, FAO, Rome, Italy, 2010, p. 378.

[2] R. H. Warning and S. W. Running, Forest Ecosystems Analysis at Multiple Scales, 3rd ed. Amsterdam, The Netherlands: Elsevier, 2007, p. 420.

[3] E. Romijn, M. Herold, L. Kooistra, D. Murdiyarso, and L. Verchot, "Assessing capacities of non-Annex I countries for national forest monitoring in the context of REDD+," Environ. Sci. Policy, vols. 19-20, pp. 33-48, May 2012

[4] K. Lim, P. Treitz, K. Baldwin, I. Morrison, and J. Green, "LiDAR remote sensing of biophysical properties of tolerant northern hardwood forests," Can. J. Remote Sens., vol. 29, no. 5, pp. 658-678, 2003.

[5] R. Nelson, W. Krabill, and G. MacLean, "Determining forest canopy characteristics using airborne laser data," Remote Sens. Environ., vol. 15, pp. 201-212, Sep. 1984.
[6] G. J. Newnham et al., "Terrestrial laser scanning for plot-scale forest measurement," Current Forestry Rep., vol. 1, no. 4, pp. 239-251, 2015.

[7] A. H. Strahler et al., "Retrieval of forest structural parameters using a ground-based LiDAR instrument (Echidna)," Can. J. Remote Sens., vol. 34 , no. 2 , pp. 426-440, 2008.

[8] D. L. B. Jupp, D. S. Culvenor, J. L. Lovell, G. J. Newnham, A. H. Strahler, and C. E. Woodcock, "Estimating forest LAI profiles and structural parameters using a ground-based laser called 'Echidna," Tree Physiol., vol. 29, pp. 171-181, Sep. 2009.

[9] W. Ni-Meister et al., "Assessing general relationships between aboveground biomass and vegetation structure parameters for improved carbon estimate from LiDAR remote sensing," J. Geophys. Res., vol. 115, p. G00E11, Oct. 2010.

[10] A. Cuni-Sanchez et al., "African savanna-forest boundary dynamics: A 20-year study," PLoS ONE, vol. 11, p. e0156934, Jun. 2016.

[11] K. Calders, J. Armston, G. Newnham, M. Herold, and N. Goodwin, "Implications of sensor configuration and topography on vertical plant profiles derived from terrestrial LiDAR," Agricult. Forest Meteorol., vol. 194, pp. 104-117, Sep. 2014.

[12] K. Calders, T. Schenkels, H. Bartholomeus, J. Armston, J. Verbesselt, and M. Herold, "Monitoring spring phenology with high temporal resolution terrestrial LiDAR measurements," Agricult. Forest Meteorol., vol. 203, pp. 158-168, Oct. 2015.

[13] K. Calders et al., "Nondestructive estimates of above-ground biomass using terrestrial laser scanning," Methods Ecol. Evol., vol. 6, no. 2, pp. 198-208, 2015.

[14] K. Calders et al., "Traceability of essential climate variables through forest stand reconstruction with terrestrial laser scanning," in Proc. SilviLaser, La Grande-Motte, France, Sep. 2015, pp. 122-124.

[15] G. Newnham et al. (2012). Evaluation of terrestrial laser scanners for measuring vegetation structure. CSIRO Sustainable Agriculture Flagship. [Online]. Available: https:// publications.csiro.au/rpr/pub?pid=csiro:EP124571

[16] M. Pfennigbauer and A. Ullrich, "Improving quality of laser scanning data acquisition through calibrated amplitude and pulse deviation measurement," Proc. SPIE, vol. 7684, p. 76841F, Apr. 2010.

[17] RIEGL. (2014). RiWAVELib: Waveform Extraction Library, accessed on Aug. 23, 2014. [Online]. Available: http//www.riegl.com

[18] RiSCAN PRO: Software Description and User's Instructions, Version 1.7.8, RIEGL LMS, 2013.

[19] A. G. Kashani, M. J. Olsen, C. E. Parrish, and N. Wilson, "A review of LiDAR radiometric processing: From ad hoc intensity correction to rigorous radiometric calibration," Sensors, vol. 15, pp. 28099-28128, Sep. 2015.

[20] A. Ullrich and M. Pfennigbauer, "Categorisation of full waveform data provided by laser scanning devices," Proc. SPIE, vol. 8186, p. 818609, Oct. 2011.

[21] RIEGL. (2013). Datasheet RIEGL VZ-400, accessed Feb. 19, 2013. [Online]. Available: http//www.riegl.com

[22] J. L. Lovell, D. L. B. Jupp, D. S. Culvenor, and N. C. Coops, "Using airborne and ground-based ranging LiDAR to measure canopy structure in Australian forests," Can. J. Remote Sens., vol. 29, pp. 607-622, Sep. 2003

[23] S. Hancock et al., "Waveform LiDAR over vegetation: An evaluation of inversion methods for estimating return energy," Remote Sens. Environ., vol. 164, pp. 208-224, Sep. 2015.

[24] W. Wagner, "Radiometric calibration of small-footprint full-waveform airborne laser scanner measurements: Basic physical concepts," ISPRS J. Photogram. Remote Sens., vol. 65, pp. 505-513, Nov. 2010.

[25] S. Kaasalainen, A. Krooks, A. Kukko, and H. Kaartinen, "Radiometric calibration of terrestrial laser scanners with external reference targets," Remote Sens., vol. 1, no. 3, pp. 144-158, 2009.

[26] L. A. Schofield, F. M. Danson, N. S. Entwistle, R. Gaulton, and S. Hancock, "Radiometric calibration of a dual-wavelength terrestrial laser scanner using neural networks," Remote Sens. Lett., vol. 7, no. 4, pp. 299-308, 2016.

[27] P. J. Hartzell, C. L. Glennie, and D. C. Finnegan, "Empirical waveform decomposition and radiometric calibration of a terrestrial full-waveform laser scanner," IEEE Trans. Geosci. Remote Sens., vol. 53, no. 1, pp. 162-172, Jan. 2015.

[28] M. Pfennigbauer, C. Wolf, and A. Ullrich, "Enhancing online waveform processing by adding new point attributes," Proc. SPIE, vol. 8731, p. 873104, May 2013.

[29] P. J. Hartzell, C. L. Glennie, and D. C. Finnegan, "Calibration of a terrestrial full waveform laser scanner," in Proc. Amer. Soc. Photogram. Remote Sens. Annu. Conf., (ASPRS), 2013, pp. 24-28. 
[30] A. Jalobeanu and G. Gonçalves, "The full-waveform LiDAR Riegl LMS-Q680I: From reverse engineering to sensor modeling," in Proc. Amer. Soc. Photogram. Remote Sens. Annu. Conf., (ASPRS), 2012.

[31] P. Pueschel, G. Newnham, and J. Hill, "Retrieval of gap fraction and effective plant area index from phase-shift terrestrial laser scans," Remote Sens., vol. 6, pp. 2601-2627, Sep. 2014.

[32] J. Hackenberg, H. Spiecker, K. Calders, M. Disney, and P. Raumonen, "SimpleTree - An efficient open source tool to build tree models from TLS clouds," Forests, vol. 6, no. 11, pp. 4245-4294, 2015.

[33] P. Raumonen et al., "Fast automatic precision tree models from terrestrial laser scanner data," Remote Sens., vol. 5, pp. 491-520, Sep. 2013.

[34] J. Liski et al., "Indirect emissions of forest bioenergy: Detailed modeling of stump-root systems," GCB Bioenergy, vol. 6, pp. 777-784, Sep. 2014.

[35] F. A. Ramirez, R. P. Armitage, and F. M. Danson, "Testing the application of terrestrial laser scanning to measure forest canopy gap fraction," Remote Sens., vol. 5, pp. 3037-3056, Sep. 2013.

[36] H. Bi, J. Turner, and M. J. Lambert, "Additive biomass equations for native eucalypt forest trees of temperate australia," Trees, vol. 18, pp. 467-479, Sep. 2004.

[37] F. M. Danson et al., "Developing a dual-wavelength full-waveform terrestrial laser scanner to characterize forest canopy structure," Agricult. Forest Meteorol., vols. 198-199, pp. 7-14, Sep. 2014.

[38] Z. Li et al., "Radiometric calibration of a dual-wavelength, fullwaveform terrestrial LiDAR," Sensors, vol. 16, p. 313, Sep. 2016.

[39] J. Armston et al., "A comparison of terrestrial laser scanning instruments for assessing forested ecosystems," in Proc. ForestSAT, vol. 2014. Riva del Garda, Italy, pp. 4-7, Nov. 2014.

[40] NASA. (2014). Missions: GEDI, accessed on Sep. 15, 2014. [Online]. Available: http://science.nasa.gov/missions/gedi/

[41] ESA. (2016). Earth Explorers-An Overview, accessed on Feb. 5, 2016. [Online]. Available: http://www.esa.int

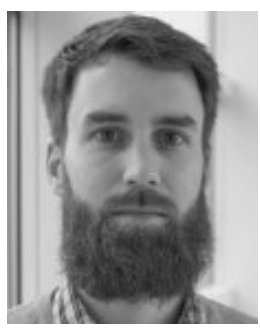

Kim Calders received the B.Sc. and M.Sc. degrees in bioscience engineering from Katholieke Universiteit Leuven, Leuven, Belgium, in 2006 and 2008, respectively, the M.Sc. degree in remote sensing from University College London (UCL), London, U.K., in 2010, and the Ph.D. degree in LiDAR remote sensing from Wageningen University, Wageningen, The Netherlands, in 2015.

Since 2015, he has been a Post-Doctoral Researcher with the National Physical Laboratory and the Department of Geography, UCL. His research interests include measurements of full 3-D vegetation structure and how this is related to airborne or spaceborne signals.

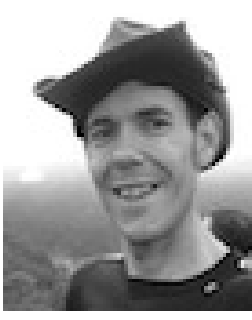

Mathias I. Disney received the B.Sc. (Hons.) degree in physics from the University of Bristol, Bristol, U.K., in 1992, the M.Sc. degree in remote sensing from the University of London, London, U.K., in 1995, and the Ph.D. degree in remote sensing from the University College London (UCL), London, in 2001.

From 2000 to 2002, he was a Lecturer with the Department of Geography, Birkbeck College, London. From 2002 to 2004, he was a Post-Doctoral Researcher with the Department of Geography, UCL, where he has been a Lecturer, a Senior Lecturer, and is currently a Reader of Remote Sensing since 2004. His research interests include 3-D measurement and modeling of vegetation, particularly forests, to characterize their structure, function and response to climate, and to develop and test new observations across scales.

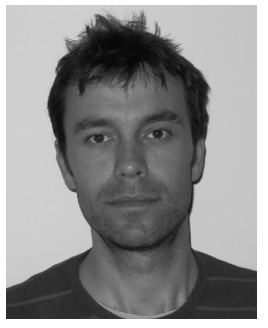

John Armston received the B.Sc. degree in biology from Macquarie University, Sydney, NSW, Australia, in 2001, and the B.Sc. (Hons.) and $\mathrm{Ph} . \mathrm{D}$. degrees in geography from The University of Queensland, Brisbane, QLD, Australia, in 2004 and 2013, respectively.

He was a Remote Sensing Scientist with the Queensland Government. He joined the Global Ecosystem Dynamics Investigation science team in 2016. His research interests include quantitative measurement and mapping of forest and woodland structure, and the development of validated satellite mapping products over large areas to support government environmental monitoring programs.

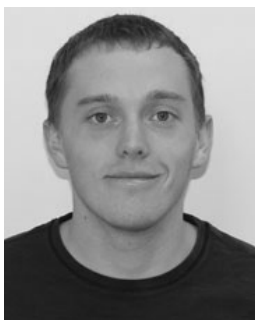

Andrew Burt received the M.Eng. degree in aerospace engineering from the University of Surrey, Surrey, U.K., in 2012. He is currently pursuing the Ph.D. degree in remote sensing with the Department of Geography, University College London, London, U.K., retrieving structural parameters of forests through terrestrial laser scanning.

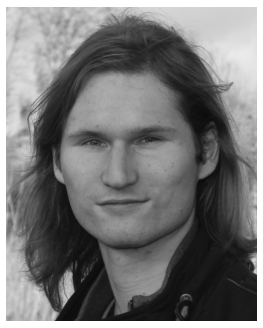

Benjamin Brede received the B.Sc. degree in geography from the Philipps Universität, Marburg, Germany, in 2012, and the M.Sc. (Hons.) degree in geoinformation science from the Wageningen University and Research Center, Wageningen, The Netherlands, in 2015, where he is currently pursuing the Ph.D. degree in vegetation remote sensing with the Laboratory of Geoinformation Science and Remote Sensing.

His research interests include vegetation parameter uct validation retrieval, multisensor data fusion, and satellite prod-

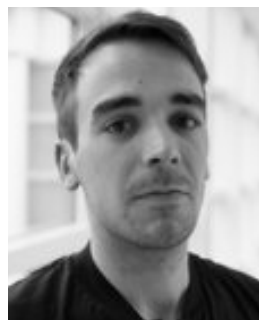

Niall Origo received the B.Sc. degree in geography from the University of Sussex, Brighton, U.K., in 2010, and the M.Sc. degree in remote sensing from University College London, London, U.K., in 2012, where he is currently pursuing the $\mathrm{Ph} . \mathrm{D}$. degree in remote sensing.

$\mathrm{He}$ is currently a Research Scientist with the National Physical Laboratory, Teddington, U.K. His research interests include the remote sensing of vegetation and the validation of vegetation remote sensing products.

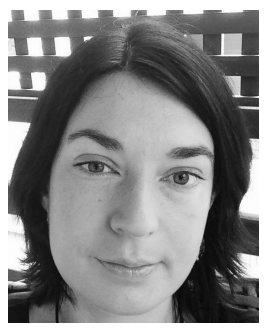

Jasmine Muir received the B.S. degree in ecology and the M.GIS. degree in remote sensing from the University of Queensland, Brisbane, QLD, Australia, in 2000 and 2009, respectively, where she is currently pursuing the $\mathrm{Ph}$.D. degree in remote sensing.

She was a Remote Sensing Scientist with the Queensland Government, Brisbane, for 11 years. She is currently a Research Fellow with the Precision Agriculture Research Group, University of New England, Armidale, NSW, Australia. Her research interests include mapping and monitoring vegetation in production and natural environments.

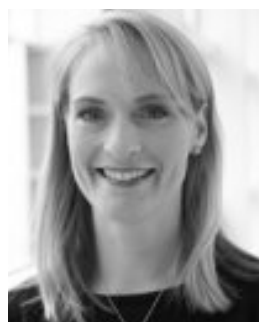

Joanne M. Nightingale received the B.Sc. (with first class Hons.) degree and the Ph.D. degree in geography from the University of Queensland, Brisbane, QLD, Australia, in 2000 and 2005, respectively.

From 2004 to 2007, she was a Post-Doctora Research Fellow at Oregon State University, Corvallis, OR, USA. From 2007 to 2013, she was a Senior Scientist with the Terrestrial Information Systems Branch, NASA Goddard Space Flight Center, Greenbelt, MD, USA. In 2013, she joined the National Physical Laboratory, London, U.K., as a Principal Research Scientist of the Earth Observation Climate and Optical Group. She currently leads a research team concerned with assessing the quality of information about the terrestrial biosphere derived from in situ measurement devices and earth observation satellites. 